\title{
Anomaly Detection Combined with Spectral Function Analysis in Hyperspectral Imagery
}

\author{
Xiaohan ZHANG, Guang YANG*, Yongbo YANG, Junhua HUANG \\ Aviation University of Air Force, Changchun, 130022, China \\ email: 15584175041@163.com
}

Keywords: Hyperspectral Imagery; Anomaly Detection; Spectral Function; Area under Spectral Profile

\begin{abstract}
To improve the time performance of hyperspectral imagery anomaly detection, this paper proposes the conception of spectral function. Using the features of spectral function such as the area under spectral profile (AUSP), potential target pixels can be separated from background pixels. Then use the data of background area to carry out anomaly detection. In this way can we save time, increase algorithm running speed, and increase the performance of original detection algorithm. At last experiment is carried to verifying the efficiency of the method proposed in this paper.
\end{abstract}

\section{Introduction}

Hyperspectral imagery has incomparable superiority in the field of targets detection and other fields owning to its high spectral resolution and rich spatial information. Anomaly detection is able to determine pixels whose signatures were spectrally distinct from background as targets of interest without any prior information. So it is becoming a hot topic in domestic and foreign hyperspectral field in recent years [1-3]. In hyperspectral imagery, each pixel represents a spectral profile. As a result, different pixels, or different surface features, have different spectral profiles. The anomaly targets have the feature of small probability of occurrence. For many targets of interest, it usually refers to the abnormal man-made objects in natural scene, so there is a big difference between the target pixel and the surrounding background pixels. As algorithm complexity is closely related to its time feature, in order to improve the algorithm performance, before carrying out anomaly detection, we usually choose to reduce the dimensionality of hyperspectral images or suppress background information to simplify the complex background and reduce computing steps.

From the point of big difference between the mathematical characteristic spectral curve of abnormal target and background, this paper proposes a new perspective anomaly detection method based on spectral characteristic profile analysis. Firstly, analyze the mathematical features of pixels such as the area under the profile. Then set the threshold to abandon the background pixels and abnormal narrowing the target may be shown. To those pixels, further anomaly detection algorithm is carried to reduce the rate of the virtual scene. As the traditional detection algorithms are based on high-dimensional matrix calculation, and mathematical characteristics profile analysis is a binary function, so the performance of reducing the amount of computation can be very good.

\section{Method of Spectral Function Characteristic Analysis}

\section{Algorithm Principle}

In hyperspectral imagery, each pixel corresponds to a spectral curve. For different pixel, its spectral characteristic is unique. Different pixel spectral profiles are shown in Figure 1.

\footnotetext{
${ }^{1}$ Fund Project: research project of “ $12^{\text {th }}$ Five-year Plan” in Department of Education of Jilin Province (NO.2015448); Science and Technology Development Program funded projects in Jilin Province (NO.20140101213JC)

Correspondence author: Guang YANG
} 


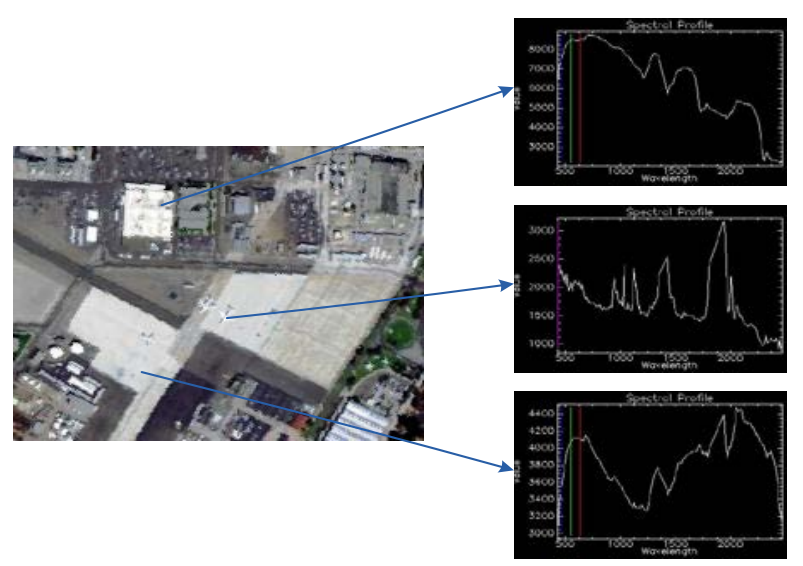

Fig.1 spectral profiles of different pixels

Traditional detection algorithms regard hyperspectral data as high-dimensional matrix. For example, for a $m \times n$ hyperspectral data with band number $L$, it can be expressed as an $m n \times L$ matrix, and each pixel is a $L \times 1$ vector. In this paper, we regard the $L \times 1$ vector as a discrete binary function. So the difference of abnormal target and background spectral profiles can turn to difference of mathematical function. By calculating the mathematical characteristics of value and then setting a certain threshold we can separate background and initial screening suspicious targets. Then those pre-selected target pixels are regarded as potential abnormal target area, while others are background areas. Use the data of backgrounds area to carry out anomaly detection, and we can get the results faster. The processing flow is shown in Figure 2.

\section{Area under Spectral Profile (AUSP)}

Suppose there is a $m \times n$ hyperspectral imagery with the band number being $L$, the spectral function of pixel of the position (i, j) $(1 \leq i \leq m, 1 \leq j \leq n)$ is $f_{(i, j)}(\lambda)$, where $\lambda$ donates for the wavelength. Ideally it should be a continuous function, but in practical applications, hyperspectral remote sensing has not yet reached a continuous band of surface features imaged, so the argument can be regarded as dimensional array, or discrete binary function.

The AUSP indicator is the area under the spectrum profile. As there is a big difference between the target and background abnormalities, different pixel spectral profile function has different AUSP value. So we can use it to as distinguish indicators. The calculating formula is shown as follows:

$$
\text { AUSP }=\sum_{k=1}^{L} f_{(i, j)}\left(\lambda_{k}\right)
$$

Figure 2 shows the calculating results using all cells index of an image:

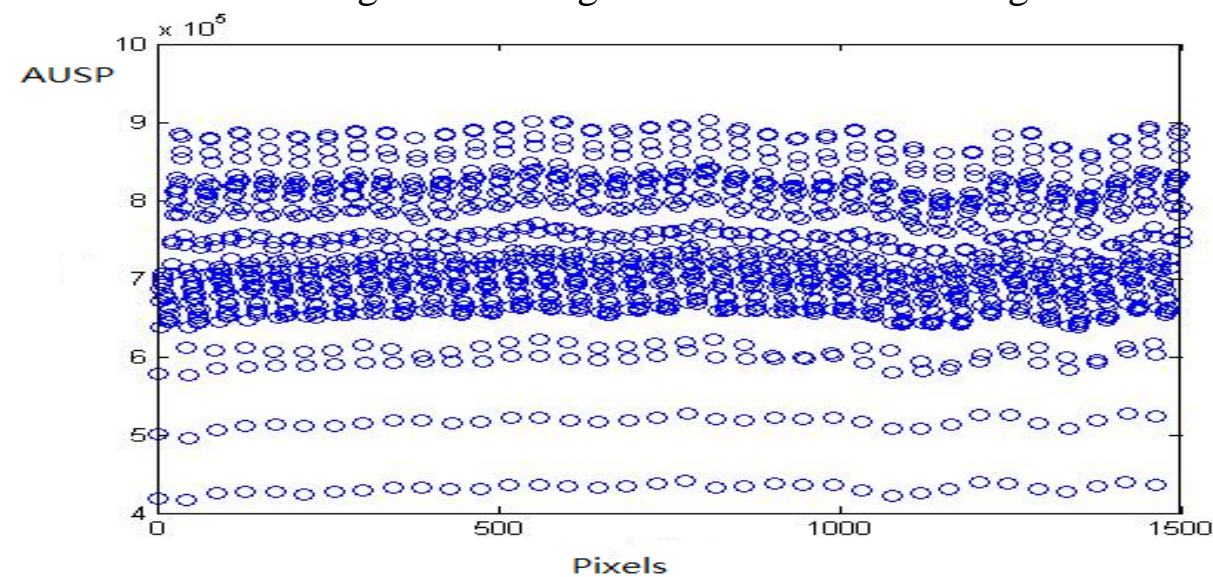

Fig.2 AUSP calculating results

From this figure, it can be seen that different pixels with different AUSP value can be preliminary isolated. Then if we select the appropriate threshold according to the figure, the region of interest can be extracted. So we can get background region and potentially anomalous target region. Next we will use background region information to detect potential anomalies. 


\section{RX Algorithm}

In hyperspectral remote sensing image anomaly detection, $\mathrm{RX}$ algorithm is the most classic. It is proposed by $\mathrm{Yu}$ and Reed in 1990[4]. RX detection algorithm assumes that the background follows Gaussian distribution, and use the maximum likelihood ratio to get the results. The algorithm is based on a pure point model and does not consider spectral mixture[5]. Each pixel only belongs to the target or the background. Usually it use a double window, including an inside and an outside window, to achieve pixel traversal detection. Suppose a hyperspectral image $X$ with $L$ bands, the imagery can be expressed as $\mathbf{X}=\left(\mathbf{x}_{1}, \mathbf{x}_{2}, \mathbf{x}_{3}, \ldots, \mathbf{x}_{\mathrm{N}}\right)$. The observation data can be expressed as:

$$
\begin{aligned}
& \mathbf{x}=\mathbf{n} ;\left(H_{0} \text { : target does not exist }\right) \\
& \mathbf{x}=\mathbf{a s}+\mathbf{n} ;\left(H_{1} \text { : target exists }\right)
\end{aligned}
$$

In the formula, $\mathbf{n}$ represents the background noise vector, $\mathbf{s}$ represents the spectral vector of target, and $a>0$. RX detection operator is:

$$
\delta_{R X}(r)=\left(r_{i}-\hat{\mu}\right)^{T} C^{-1}\left(r_{i}-\hat{\mu}\right)
$$

In this formula, $r_{i}$ is observation spectral, $\mu^{\$}$ is the estimated background mean vector, and $C^{-1}$ is the estimated background covariance matrix.

\section{RX algorithm combining AUSP analysis}

RX algorithm needs to obtain background information of the image. The traditional method uses the double window detector to pixels to obtain background information between the inner window and the outside window. As the information of the pixels being background or target is unknown, there may be the cases of false alarm or missing true targets. If you can accurately extract the appropriate background information from potential targets, we can improve the accuracy and efficiency of detection.

After the AUSP index of image has been computed, basic information has been separated, and the background area can be divided from potentially anomalous target area. Here to reduce the amount of calculation, only the potential abnormal cell target area is detected. When these pixels were in RX double window traversal, it is considered as background and serves for the computing of background mean and covariance matrix. At this time may appear some cell detection window phenomenon of potential targets and they are all located in areas of potential targets, and if more than half of the pixel area is not in the background, then the detection window for general detection of RX is carried to reduce virtual false.

\section{Experiment Result}

\section{Experiment Protocol}

Firstly, the AUSP index of image is operated, and the threshold value can be obtained by dividing the target area from the background area. Then, the image segmentation algorithm of A-RX method proposed in this paper is used to obtain results shown in Fig. 3. Besides, the original $\mathrm{RX}$ algorithm is also used to the same image for analysis, and the two results of anomaly detection methods were compared to prove the effectiveness of method in this paper.

\section{Data Used in the Experiment}

The experimental data used in this paper is the hyperspectral image gotten by AVIRIS imaging spectrometer in the United States on a US Naval Air Base. The data has been preprocessed, removing the serious noise pollution and water vapor absorption bands. And remaining 189 bands are used in the experiment. the size of the image is $100 \times 100$. The $7^{\text {th }}$ band and ground targets distribution are shown in Fig. 3. 


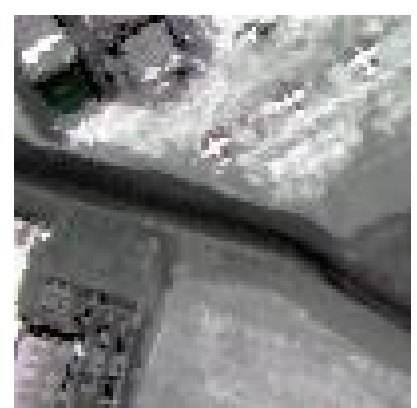

(a) The seventh band

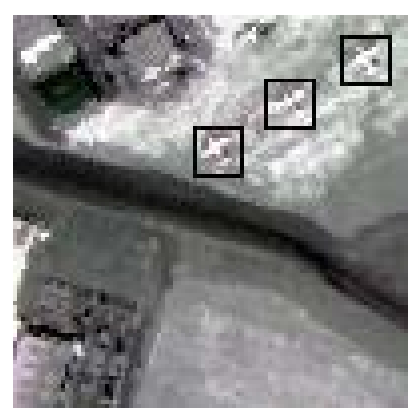

(b) Ground target distribution

\section{Test results and analysis}

Fig.3 The seventh band and its ground target distribution

First, experimental data were normalized, and then each pixel characteristic value calculation performed AOSP give AOSP each pixel value distribution shown in Figure 4. Removing dense distribution interval of pixels to obtain background and potential anomaly separation is shown in Figure 5. Fig. 5 has been removed 6225 background pixels, and Fig. 8 target distribution criteria diagram comparison can be found, the basic form of the three aircrafts have been shown, but there are still a large number of virtual resort. When RX detection in progress, within the window size is selected as the $3 \times 3$, outside the window size of $9 \times 9$

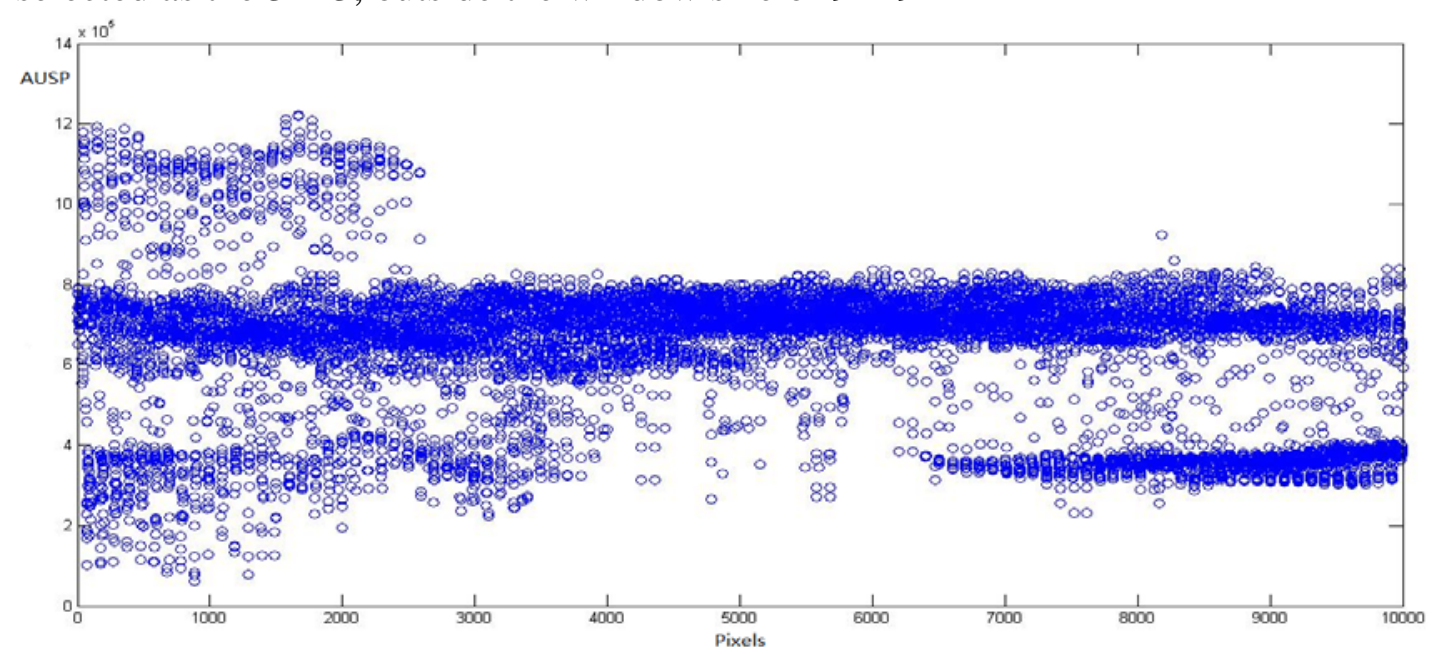

Fig.4 AUSP index distribution of the imagery

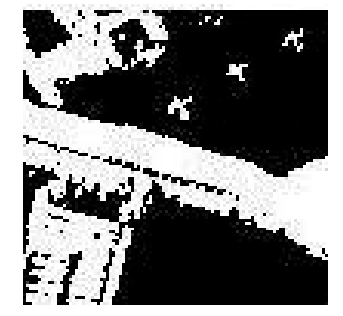

Fig.5 potential anomalies and background distribution

The standard image of the target is shown as follows:

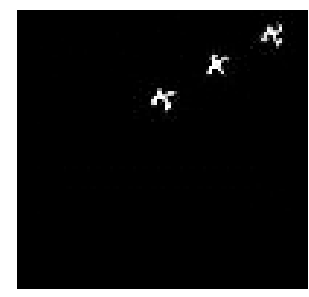

Fig.6 standard target image

The results is shown as Fig.7.

To illustrate the effecttiveness of the method, in Table 1 lists the original RX algorithm under the same detection threshold detected target number, the number of pixels of the target pixel share, 
the share of false alarms the number of pixels, and cited four time method used. Specific results are shown in Table 1. As can be seen from the table, the effect of KRX detection algorithm and its improved algorithm is much better than the RX algorithms, this algorithm works best. However, the detection time, detection algorithms using KRX time was used for a redundant RX algorithms because KRX algorithm is a more complex calculation algorithms can not be used for real-time detection. However, the algorithm used time have less time than P-KRX algorithm used to suppress background is superior to other methods to improve detection effectiveness.

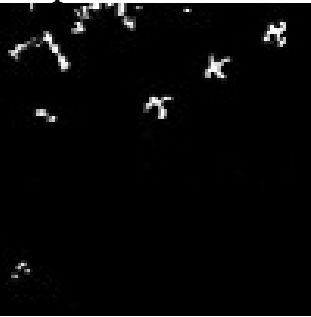

a) result using the method of this paper

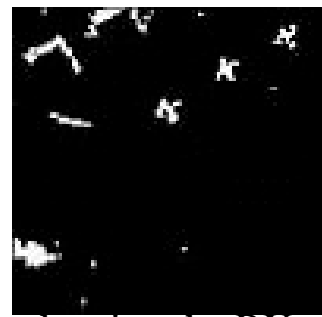

b) result using the $\mathrm{RX}$ method

fig.7 experiment results

Tab.1 Algorithm performance comparison of four methods

\begin{tabular}{ccccc}
\hline & $\begin{array}{c}\text { Target } \\
\text { number }\end{array}$ & Target pixels & False pixels & time \\
\hline RX algorithm & 3 & 72 & 199 & $28.1278 \mathrm{~s}$ \\
A-RX algorithm & 3 & 60 & 131 & $21.0657 \mathrm{~s}$ \\
\hline
\end{tabular}

\section{Conclusions}

This paper presents a new method for detecting abnormal pretreatment detected prior to the abnormal image is separated into a background region and a preliminary abnormal potential target area. This method is effective to improve the effectiveness and efficiency of hyperspectral detection anomaly detection. At the same time, this method is also proposed to simplify the hyperspectral image anomaly detection provides a new idea and method, the study is significant anomaly detection based on hyperspectral Eliminate background.

\section{Reference}

[1] Skauli T, Haavardsholm T, Kasen I, et al. Hyperspectral imaging technology and systems, exemplified by airborne real-time target detection[C]. 2011 Conference on L-asers andElectro-Optics (CLEO), 2011.5:1-6.

[2] Reed I S, Yu X. Adaptive multiple-band CFAR detection of an optical pattern with unknown spectral distribution[J]. Acoustics, Speech and Signal Processing, IEEE Transactions on, 1990, 38(10): 1760-1770.

[3] TITS L, SOMERS B, COPPIN P. The potential and limitations of a clustering approach for the improved efficiency of multiple endmember spectral mixture analysis in plant production system monitoring[J]. IEEE. Transactions on Geoscience and Remote Sensing, 2012, 50(6): 2273-2286.

[4] Safa Khazai, Saeid Homayouni, Abdolreza Safari. Anomaly Detection in Hyperspectral Images Based on an Adaptive Support Vector Method[J]. IEEE Geoscience and Remote Sensing Letters, 2011, 8(4):646-650.

[5] CHENG Baozhi, ZHAO Chunhui, WANG Yulei. Anomaly detection of hyperspectral image for band subsets based on fourth order cululant[J]. Journal of Optoelectronics.Laser, 2012, 23(8): 1582-1588. 